Check for updates

Cite this: RSC Adv., 2017, 7, 38565

\title{
Direct writing of a conducting polymer pattern in aqueous solution by using an ultrashort laser pulse $\uparrow$
}

\author{
Neha Agarwal, ${ }^{a}$ Hyobong Ryu, (D) a Melanie Mangang, ${ }^{b}$ Wilhelm Pfleging ${ }^{b}$ \\ and Jungtae $\mathrm{Kim}^{\star \mathrm{a}}$
}

A new method for the polymerization and patterning of a conducting polymer by using a femtosecond laser is presented. In this research, pyrrole-3-carboxylic acid (PCA), an acid derivative of pyrrole, was used because of its higher water solubility and favorable adhesion to the glass surface. The PCA pattern was fabricated in a microfluidic channel between gold electrodes and comprised micro-/nano structures. The optimized parameters of the femtosecond laser were investigated for obtaining sharp and defined conducting polymer patterns. The aqueous state of the polymerization enhanced the possibility of applying additional dopants for extending the functionality of the pattern, including embedding of bio molecules. The generated patterns of the conducting polymer were observed and evaluated by atomic force microscopy and conductivity measurements.

Received 8th May 2017

Accepted 31st July 2017

DOI: 10.1039/c7ra05195f

rsc.li/rsc-advances utilized as the advancement in the field of polymerization and structuring due to the following advantages; ${ }^{13,14}$ DLW has several advantages of high precision structures, easy fabrication of structures, fast and relatively reproducible patterns without a photomask. With the utilization of the ultrashort pulses, nonlinear optical processes such as two-photon absorption can take place. The structure formations are three dimensional in micro/nano scale.

Furthermore, the DLW can be adequately utilized in aqueous condition, which is the most favorable for biological materials. The conventional chemical and electrochemical polymerization techniques in aqueous solution is slow and this long polymerization time leads to irregular and unstable solidification generating inaccurate structures. ${ }^{15}$ Whereas DLW of the aqueous solution to fabricate sub-micron structures on the substrate is advantageous as the electron flow for polymerization can be maintained local only in the interested area. Therefore, the writing area is only the focal area and the structures are formed in the focal plane. ${ }^{12}$ Also, the adhesion of the polymer layer to the substrate is one of the advantages of the DLW process due to the varied emission wavelengths depending on the mechanism used on the photon generation. ${ }^{16}$ Since, the monomers can be prepared in different concentrations to form the aqueous solution; there is a possibility of applying additional dopants in order to enhance the functionalities of the material. ${ }^{17}$ In this work, we have performed pyrrole polymerization in aqueous solution by using direct fs laser writing technique. In order to conduct the process, a microfluidic chip has been fabricated which has gold electrodes in a microfluidic channel on a quartz glass substrate (Fig. 1(a)). The polymerization was done in the gap between the gold electrodes. The aqueous mixture solution was injected into the microfluidic
${ }^{a}$ Korea Institute of Science and Technology Europe GmbH, Campus E7 1, 66123 Saarbrücken, Germany. E-mail: tais@kist-europe.de

${ }^{b}$ Applied Material Physics, Karlsruhe Institute of Technology, Hermann-von-Helmholtz Platz 1, 76344 Eggenstein-Leopoldshafen, Germany

$\dagger$ Electronic supplementary information (ESI) available. See DOI: 10.1039/c7ra05195f 
(a)

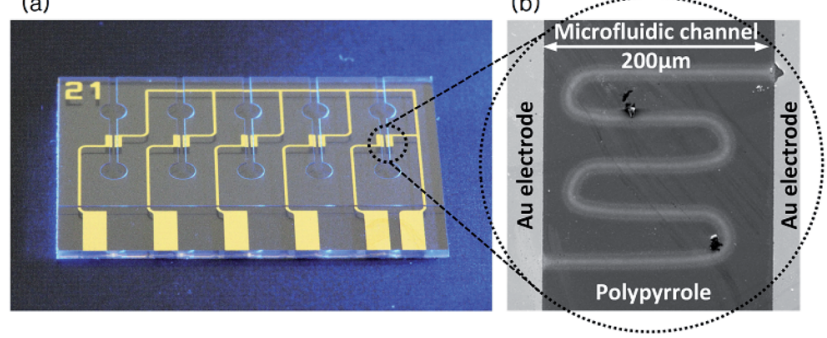

Fig. 1 (a) Image of the fabricated microfluidic chip. Each channel has $200 \mu \mathrm{m}$ width and $100 \mu \mathrm{m}$ thickness made by SU-8 dry film on quartz glass wafer. (b) Scanning electron microscopy image of polypyrrole pattern. It was written by the laser between the gold electrodes.

channel and the laser beam was focused onto the glass surface adjacent to the gold electrode edge for the polymerization to take place.

The pattern has been drawn using the laser to form micro-/ nano structures of the conductive polymer as shown in Fig. 1(b). After the polymerization, conductivity and surface topology of the structure were measured and analyzed.

\section{Materials and methods}

\section{Materials}

Pyrrole-3-carboxylic acid (PCA) of analytical grade was purchased from Sigma-Aldrich. (3-Aminopropyl) triethoxysilane and $N$-(3-triethoxysilypropyl)-4-hydroxybutyramide, i.e., both the different types of silanes were used for the silanization of the surfaces and were bought from Sigma-Aldrich. The polyamidoamine (PAMAM) G4.0 dendrimers were obtained via Sigma-Aldrich Chemie GmBH from Dendritech Inc. (USA). A $100 \mathrm{mM}$ aqueous solution of PCA was prepared from dissolving of $22 \mu \mathrm{g}$ of PCA in $2 \mathrm{~mL}$ of distilled water. A dendrimer mixed solution was prepared with 50\% (vol\%) mixture of PAMAM G4.0 dendrimers and PCA.

\section{Microfluidic chip}

The quartz glass, $700 \mathrm{~nm}$ thick, was cleaned by piranha solution. After the rinsing step with using de-ionized (DI) water, $10 \mathrm{~nm} \mathrm{Cr}$ as adhesion layer, and $20 \mathrm{~nm}$ Au layer were deposited by magnetron sputtering. The electrode pattern was defined by optical lithography using positive photoresist and subsequent wet etching process with a mixture of $\mathrm{KI}: \mathrm{I}_{2}: \mathrm{DI}$ water ( $4: 1: 40)$ to remove the $\mathrm{Au}$, and a dedicated chromium etchant. Finally, micro channel structures have been fabricated by using $100 \mu \mathrm{m}$ thick SU-8 dry film and photolithography processes on the quartz glass wafer.

\section{Methods}

The microfluidic chips were cleaned in an ultrasonic bath for 5 minutes using isopropanol and then dried with nitrogen. Oxygen plasma treatment was applied for 3 minutes with the power of $70 \mathrm{~W}$. In order to get $-\mathrm{OH}$ or $-\mathrm{NH}_{2}$ terminated groups on the quartz glass surface, 2\% $N$-(3-triethoxysilypropyl)-4- hydroxybutyramide or (3-aminopropyl) triethoxysilane was prepared in toluene and the chips were dip-casted for 30 minutes in an oven at $80{ }^{\circ} \mathrm{C}$ respectively. After rinsing with toluene and drying with nitrogen, the chips were cured at $100{ }^{\circ} \mathrm{C}$ in an oven for an hour.

Conducting polymer patterns were fabricated by using a micromachining workstation (PS450-TO, Optec, Belgium) equipped with an ultrafast fiber laser (Tangerine, Amplitude Systems, France) in Karlsruhe Nano Micro Facility. It is operated with an average power of $20 \mathrm{~W}$ and maximum pulse energy of $100 \mu \mathrm{J}$ at $1030 \mathrm{~nm}$ (TEMOO with $\left.M^{2}<1.3\right)$. The pulse duration can be tuned in the range from 350 fs up to $10 \mathrm{ps}$ and the wavelength can be switched from fundamental $(1030 \mathrm{~nm})$ to 2nd $(515 \mathrm{~nm})$ or $3 \mathrm{rd}(343 \mathrm{~nm})$ harmonics. For the polymerization study, the $343 \mathrm{~nm}$ wavelength was chosen with a laser repetition rate of $200 \mathrm{kHz}$ and a spot size of about $20 \mu \mathrm{m}$ (see ESI: Fig. S2†). The pulse duration was set to $400 \mathrm{fs}$. The laser beam was scanned over the sample surface using a RhothorTM Laser Deflection Systems scan head (Newson Engineering BV, Belgium). The process was performed by scanning the laser beam using a f-theta lens with a focal length of $100 \mathrm{~mm}$. The chip was flipped over and mounted on the XYZ piezo stage. The laser beam was focused through the $700 \mu \mathrm{m}$ quartz glass substrate into the aqueous solution which was injected in the microfluidic channel. The laser movement and the focusing on the sample were controlled by the software of the laser system. Several laser and process parameters such as laser power, writing speed, number of writing repetitions and focal position were tested.

A phase contrast microscope was employed in order to take images of the polymerized pattern. Atomic force microscopy was also done with a Park Systems NX10 AFM equipped with a non-contact mode probe (PPP-NCLR-10, Nanosensors). The conductivity of the patterns was measured using Keysight B1500A Semiconductor Device Analyzer.

\section{Results and discussion, experimental}

The results of laser polymerization were dependent on several factors comprising of surface type of the substrate, different aqueous mixture solutions including concentration variations and the laser parameters. A number of tests have been performed to fabricate fine and sharp pattern from polymerized PCA. The adhesion between a deposited polymer and a substrate is mainly determined by the interfacial properties of the substrate. In this work, the adhesion property has been tested by two different surface modifications. (3-Aminopropyl) triethoxysilane (APTES) and $N$-(3-triethoxysilypropyl)-4hydroxybutyramide were used introducing $-\mathrm{NH}_{2}$ and $-\mathrm{OH}$ groups on the quartz glass surface respectively for enhancement of the adhesion. Several preliminary tests were performed for the optimization of the surface properties suitable for the polymerization. It was found that the $-\mathrm{OH}$ terminated groups showed relatively uniform adhesion along the laser writing path whereas the $-\mathrm{NH}_{2}$ terminated groups led to strong but scattered and radical adhesion (see ESI: Fig. S3†). 
During the laser polymerization process, various parameters of the laser were tested whereas the speed of the laser beam was always maintained at $100 \mathrm{~mm} \mathrm{~s}^{-1}$. We selected low power that could derive polymerization with maximum 1000 repetitions suppressing possible damage of biological molecules in future applications. The laser power was varied between $0.3-7 \mathrm{~mW}$ (34-40\% of the laser power).

However, maximum number of repetitions was kept low during the tests with laser power of $2 \mathrm{~mW}$ or higher because, for those laser power and high number of repetitions, the surface between the gold electrodes showed no pattern along the laser path whereas the gold electrodes were obviously damaged by the laser.

For the comparison of the results, writing by the laser power of $0.3 \mathrm{~mW}$ and $0.6 \mathrm{~mW}$ were tested with several writing repetitions such as 1000, 750, 500, 250 and 100 and the microscopic images were taken as follows.

Fig. 2 shows the polymerized structures between the gold electrodes from a laser power of $0.3 \mathrm{~mW}$ and $0.6 \mathrm{~mW}$ with various writing repetitions. The image was taken by phase contrast microscope after isopropanol washing and drying. As shown in Fig. 2, the conducting polymer structure with $0.6 \mathrm{~mW}$ laser power shows more precisely defined structure than 0.3 $\mathrm{mW}$ and can be formed well as repetitions number increase.

However, the conductivity results show that polymerization induced by a laser power of $0.3 \mathrm{~mW}$ leads to a higher conductivity in comparison to the results of $0.6 \mathrm{~mW}$ power. A comparative data of the conductivity measurements are shown in Fig. 3. Patterns on hydroxyl group terminated surface showed higher conductivity in overall. The conductivity of max. $17 \mathrm{~S}$ $\mathrm{cm}^{-1}$ has been obtained from PCA polymer pattern with $0.3 \mathrm{~mW}$ laser power and 750 laser writing repetition. The patterns with a higher laser power $(0.6 \mathrm{~mW})$ showed low conductivity although the pattern appearance was finer.

Fig. 4 shows AFM analysis results from one of the curved part of the pattern. The polymerized pattern shows that the width of the structure is around $18 \mu \mathrm{m}$ in accordance with diameter of the laser spot. However, only the area of 7-8 $\mu$ m width along the
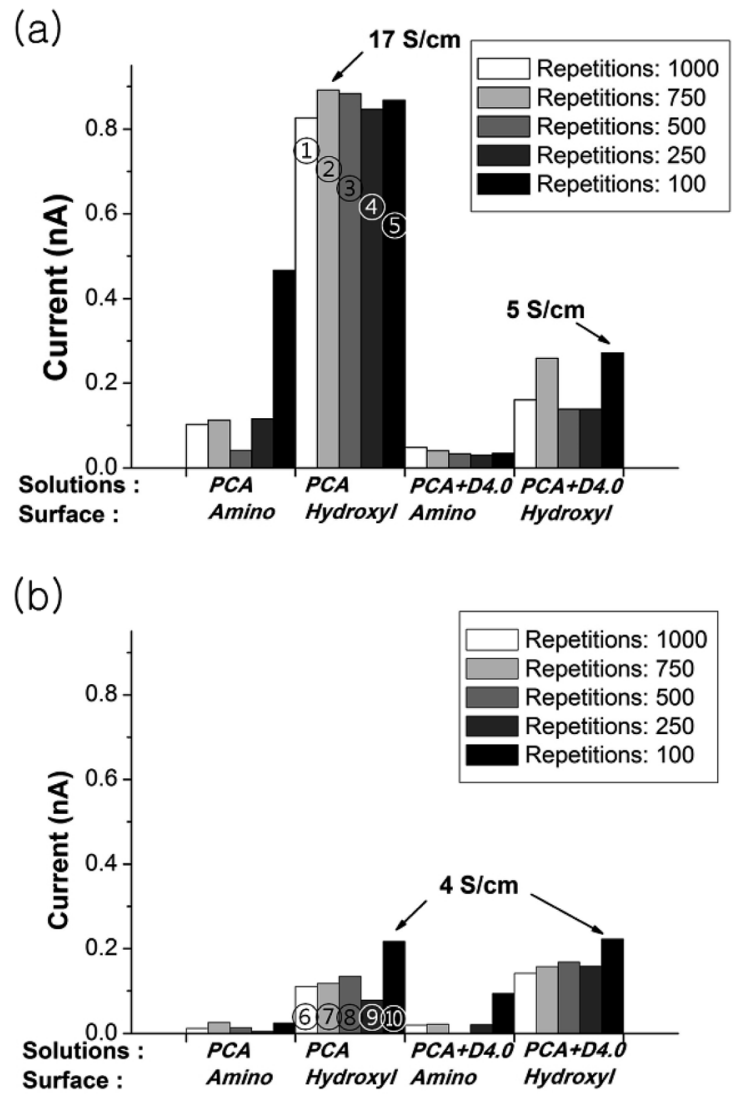

Fig. 3 Conductivity comparisons of fabricated patterns by various parameters. The current is measured at $2 \mathrm{~V}$ and laser power is (a) 0.3 $\mathrm{mW}$ and (b) $0.6 \mathrm{~mW}$. (1)-(10) are denoted to the samples of Fig. 2.

center line shows higher density and height of $113 \mu \mathrm{m}$ by 0.3 $\mathrm{mW}$ laser power with 1000 writing repetitions from the PCA solution. This type of profile has been obtained due to the Gaussian nature of the femtosecond laser intensity.

A mixture of PAMAM G4.0 dendrimer and PCA has been used to demonstrate direct functionalization of the conductive pattern by applying dopants. The dendrimer can be
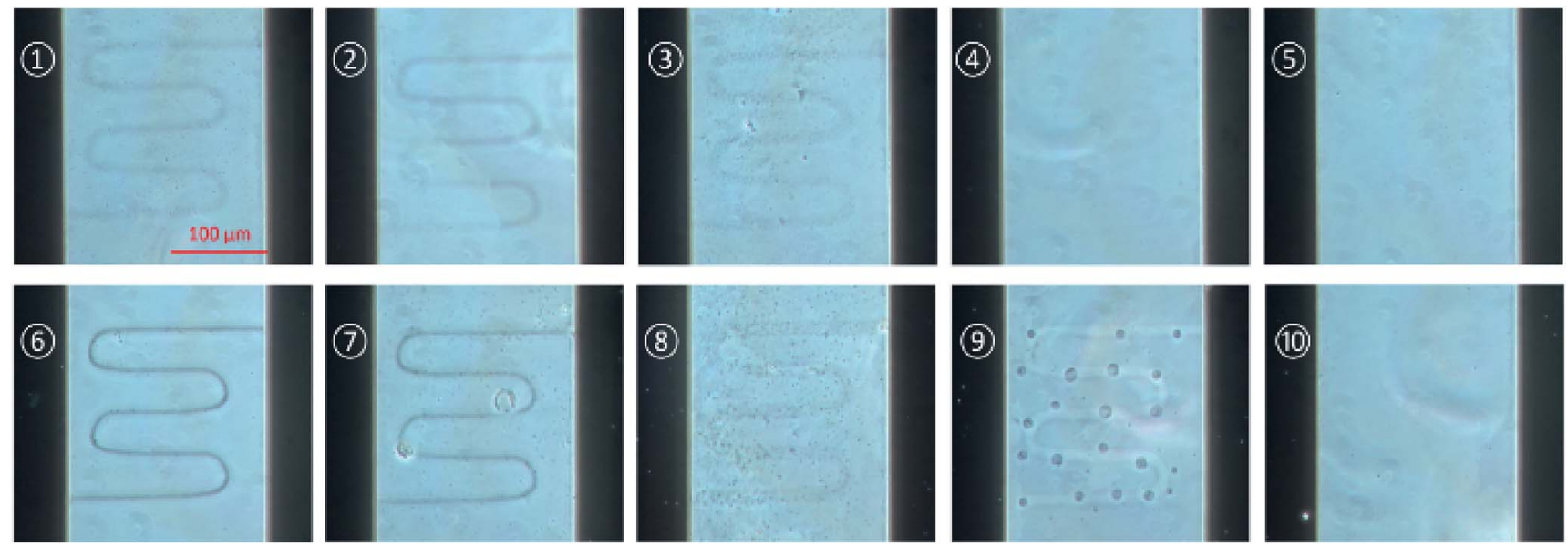

Fig. 2 Microscopic images of the polymerized PCA patterns with various writing repetitions, (1), (6) 1000, (2), (7) 750, (3), (8)) 500, (4), (9) 250, (5), (10) 100. The images of (1)-(5) are from $0.3 \mathrm{~mW}$ laser power experiment and (6)-(10) are from $0.6 \mathrm{~mW}$. 

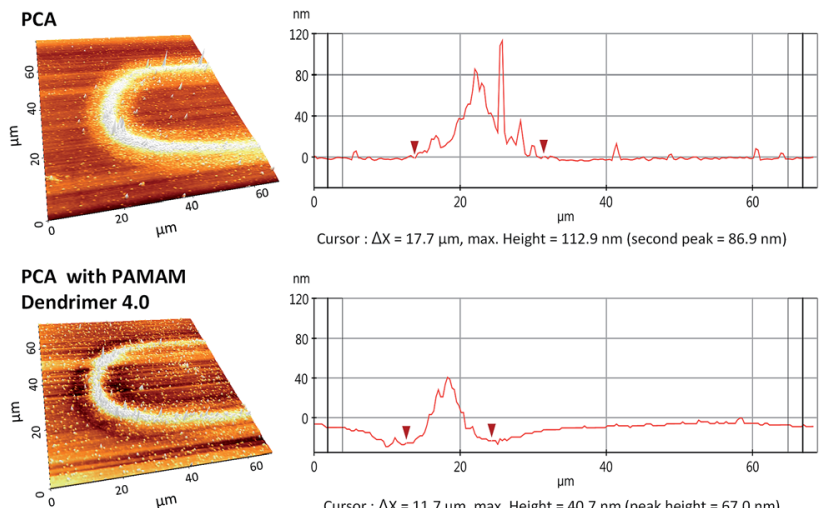

Cursor: $\Delta x=11.7 \mu \mathrm{m}, \max$. Height $=40.7 \mathrm{~nm}$ (peak height $=67.0 \mathrm{~nm}$

Fig. 4 AFM measurements of laser generated conductive PCA polymer patterns. Curved parts of the patterns were scanned in the noncontact mode. Cross sectional profiles (right) were measured in the middle of the curved path.

incorporated with PCA polymer and embedded with specific moiety for active biological targets.

Comparing results from the PCA solution and the PAMAM G4.0 dendrimer mixture, the width of the pattern shows considerably reduced height of $40 \mathrm{~nm}$ as well as the reduced width of around $12 \mu \mathrm{m}$. The addition of dendrimer led to increased absorption of the laser power and showed the possibility to control the dimensions of the structure. Several different laser and process parameters were investigated for different types of aqueous mixtures of PCA such as phosphate buffer ( $\mathrm{pH}$ 7.0) in order to optimize the parameters suitable for the application as a bio-sensor. Phosphate buffer is a medium in which biological molecules stay stable and does not denaturate from its original form.

\section{Conclusions}

A direct fs laser writing technique has been employed for the patterning process rather than the conventional lithography or fused deposition techniques. Conducting polymer patterns have been obtained from the aqueous mixture solution in the middle of the microfluidic channels. Several processing parameters such as laser power, repetitions of writing as well as surface modification of the glass substrate have been tested. Using an aqueous solution and a microfluidic chip, the presented method showed the possibility for direct fabrication of a sensor element with biological molecules. Although further studies and evaluations are required, the results from the fs laser method showed the possibility of having a biological molecule integrated into the pattern using a PCA solution.

\section{Acknowledgements}

We acknowledge KIST Europe Basic fund project 11512 for the financial support. The laser structuring work has received funding from the European Union's Horizon 2020 research and innovation program under the Marie Sklodowska-Curie grant agreement no. 644971. Finally, the support for laser processing by the Karlsruhe Nano Micro Facility (KNMF, http:// www.knmf.kit.edu/) a Helmholtz research infra-structure at the Karlsruhe Institute of Technology (KIT) is gratefully acknowledged.

\section{References}

1 B. Adhikari and S. Majumdar, Prog. Polym. Sci., 2004, 29(7), 699-766.

2 S. Roy and Z. Gao, Nano Today, 2009, 4, 318-334.

3 A. Mulchandani and N. V. Myung, Conducting polymer nanowires-based label-free biosensors, Curr. Opin. Biotechnol., 2011, 22, 502-508, DOI: 10.1016/ j.copbio.2011.05.508.

4 A. Gelmi, M. J. Higgins and G. G. Wallace, Physical surface and electromechanical properties of doped polypyrrole biomaterials, Biomaterials, 2010, 31, 1974-1983, DOI: 10.1016/j.biomaterials.2009.11.040.

5 Z. Niu, M. a. Bruckman, B. Harp, C. M. Mello and Q. Wang, Bacteriophage M13 as a scaffold for preparing conductive polymeric composite fibers, Nano Res., 2008, 1, 235-241, DOI: $10.1007 / \mathrm{s} 12274-008-8027-2$.

6 K. C. Donavan, J. A. Arter, G. A. Weiss and R. M. Penner, Virus-Poly(3,4-ethylenedioxythiophene) biocomposite films, Langmuir, 2012, 28, 12581-12587, DOI: 10.1021/la302473j.

7 J. A. Arter, D. K. Taggart, T. M. McIntire, R. M. Penner and G. A. Weiss, Virus-PEDOT nanowires for biosensing, Nano Lett., 2010, 10, 4858-4862, DOI: 10.1021/nl1025826.

8 J. a. Arter, J. E. Diaz, K. C. Donavan, T. Yuan, R. M. Penner and G. a. Weiss, Virus-polymer hybrid nanowires tailored to detect prostate-specific membrane antigen, Anal. Chem., 2012, 84, 2776-2783, DOI: 10.1021/ac203143y.

9 A. Malinauskas, J. Malinauskiene and A. Ramanavičius, Conducting polymer-based nanostructurized materials: electrochemical aspects, Nanotechnology, 2005, 16, R51R62, DOI: 10.1088/0957-4484/16/10/R01.

10 A. Ramanavicius, A. Ramanaviciene and A. Malinauskas, Electrochemical sensors based on conducting polymerpolypyrrole, Electrochim. Acta, 2006, 51, 6025-6037, DOI: 10.1016/j.electacta.2005.11.052.

11 M. F. Attia, T. Azib, Z. Salmi, A. Singh, P. Decorse, N. Battaglini, H. Lecoq, M. Omastová, A. A. Higazy, A. M. Elshafei, M. M. Hashem and M. M. Chehimi, Onestep UV-induced modification of cellulose fabrics by polypyrrole/silver nanocomposite films, J. Colloid Interface Sci., 2013, 393, 130-137, DOI: 10.1016/j.jcis.2012.11.008.

12 S. D. Gittard and R. J. Narayan, Laser direct writing of microand nano-scale medical devices, Expert Rev. Med. Devices, 2010, 7, 343-356, DOI: 10.1586/erd.10.14.

13 C. Breck Hitz, J. J. Ewing and J. Hecht, Introduction to Laser Technology, Wiley-IEEE Press, 4th edn, 2014, ISBN: 978-0470-91620-9.

14 K. Ke, E. F. Hasselbrink and A. J. Hunt, Rapidly prototyped three-dimensional nanofluidic channel networks in glass substrates, Anal. Chem., 2005, 77, 5083-5088, DOI: 10.1021/ ac0505167. 
15 T. N. Kim, K. Campbell, A. Groisman, D. Kleinfeld and C. B. Schaffer, Femtosecond laser-drilled capillary integrated into a microfluidic device, Appl. Phys. Lett., 2005, 86, 1-3, DOI: 10.1063/1.1926423.

16 J. Sone, K. Yamada, A. Asami and J. Chen, Sub-Micrometer Size Structure Fabrication Using a Conductive Polymer, Micromachines, 2014, 6, 96-109, DOI: 10.3390/mi6010096.
17 Y. H. Lee, J. Y. Lee and D. S. Lee, A novel conducting soluble polypyrrole composite with a polymeric co-dopant, Synth. Met., 2000, 114, 347-353, DOI: 10.1016/S0379-6779(00) 00268-X. 